\title{
De l'imagerie médicale au renversement temporel
}

Mathias Fink

\section{(2) OpenEdition}

Journals

Édition électronique

URL : https://journals.openedition.org/histoire-cnrs/9096

DOI : 10.4000/histoire-cnrs.9096

ISSN : 1955-2408

Éditeur

CNRS Éditions

Édition imprimée

Date de publication : 5 octobre 2009

ISSN : 1298-9800

Référence électronique

Mathias Fink, " De l'imagerie médicale au renversement temporel », La revue pour l'histoire du CNRS [En ligne], 24 | 2009, mis en ligne le 05 octobre 2009, consulté le 20 mai 2021. URL : http://

journals.openedition.org/histoire-cnrs/9096; DOI : https://doi.org/10.4000/histoire-cnrs.9096

Ce document a été généré automatiquement le 20 mai 2021

Comité pour l'histoire du CNRS 


\title{
De l'imagerie médicale au renversement temporel
}

\author{
Mathias Fink
}

1 La revue pour l'histoire du CNRS.Quel a été votre cheminement intellectuel et scientifique?

2 Mathias Fink. J'ai sans doute un trajet atypique : j'ai commencé par la physique du solide, je me suis ensuite intéressé à l'archéologie sous-marine puis à l'imagerie médicale. Dans les années 1970, j'ai connu les débuts de l'imagerie médicale et l'essor des ultrasons. J'ai été consultant chez Philips. Pendant 19 ans, je me suis frotté au monde de l'industrie tout en restant dans le milieu académique. J'observais les méthodes, les réussites et les échecs.

La revue... Dans votre carrière de physicien, le transfert technologique revient comme un leitmotiv. Nul ne peut contester votre fibre entrepreneuriale. Pourtant, vous n'avez jamais réellement franchi le pas.

M. F. Je ne suis pas un industriel, je suis un inventeur ! Les inventeurs ont leur place au CNRS, pas les industriels! Ce qui m'intéresse, c'est faire de la belle physique, créer, fabriquer des appareils à partir des inventions, les tester. Je sens quand « le jeu en vaut la chandelle ", mais je n'ai pas les compétences pour créer une société, ni l'âme d'un salarié d'entreprise mais plutôt celle d'un créateur d'emplois pour des chercheurs qui n'hésiteraient pas eux, à passer de l'autre côté de la barrière.

5 La revue... Les conclusions en 1998 du rapport de mission d'Henri Guillaume sur la technologie et l'innovation en France étaient plutôt pessimistes' ${ }^{1}$. Le paysage de l'entreprise a-t-il changé en 10 ans, avec notamment la loi sur l'innovation de 1999 ?

6 M. F. J'ai participé à la création de pas mal d'entreprises! Mais elles n'ont pas toujours résisté, parce que la loi sur l'innovation, qui constitue un véritable progrès, n'existait pas encore ! J'ai soutenu la création de US Control en 1990 à partir de notre laboratoire. Faute d'aide publique, cette société a été rachetée par une boîte canadienne, devenue le leader mondial en contrôle non destructif par ultrasons. Fabrice Cancre que j'ai formé, 
dirige désormais Olympus à Boston. Cette société n'avait aucune chance de réussir avant 1999. Aujourd'hui, on procéderait différemment.

7 La revue... Le nécessaire accompagnement à la création d'entreprise relève du rôle de " passeurs » entre les mondes académique et économique.

Dans un contexte fortement concurrentiel, un chercheur, aussi «accompli» et chevronné soit-il, peut-il s'improviser chef d'entreprise ? Quel rôle joue le CNRS ?

M. F. Dépositaire et détenteur des brevets, le CNRS peut accorder ou non une licence exclusive à une société pour développer le produit. Il peut en même temps vendre définitivement le brevet, récupérer des royalties ou devenir actionnaire de la dite société. Pour qu'une société puisse croître de façon significative, elle doit posséder ses propres brevets sans quoi les investisseurs ne s'engagent pas.

La revue... Vous avez réussi à surmonter beaucoup d'obstacles et de nombreuses entreprises ont été créées à partir de votre laboratoire.

11 M. F. Après la loi sur l'innovation, la première entreprise, Echosens, créée à partir du laboratoire, a vu le jour en 1990. Elle a développé un appareil «le fibroscan », mis au point au laboratoire, qui mesure le degré de fibrose du foie. Cette entreprise prospère emploie une quarantaine de personnes avec un chiffre d'affaire de 10 millions d'euros. Puis en 2003, Sensitive Object a été créée par Ros Kiri Ing, maître de conférences et chercheur du laboratoire parti en détachement pour l'occasion. Cette société développe des écrans tactiles et des objets intelligents à partir du concept des miroirs acoustiques à retournement temporel. Puis Supersonic Imagine, entreprise d'imagerie et de thérapie médicale, a vu le jour en 2005 et nous avons créé dernièrement une entreprise dans le domaine des télécommunications Time Reversal Communications.

La revue... Supersonic Imagine vous tient particulièrement à cœur.

M. F. En effet, cette entreprise s'est lancée, à partir de nos recherches, dans la fabrication d'un appareil d'imagerie médicale tout à fait révolutionnaire et dans la mise au point d'un appareil de thérapie par ultrasons pour détruire à distance des tumeurs dans le cerveau. J'ai réussi à convaincre Jacques Souquet qui était aux États-Unis depuis 22 ans de revenir en France. Il y dirigeait la R\&D de Philips Medicals System. Il a rapatrié onze "cerveaux» français qui avaient été en partie formés par nous et Supersonic Imagine emploie aujourd'hui près de 100 personnes. Les capitaux-risqueurs ont investi dès le départ 10 millions d'euros ce qui était tout à fait exceptionnel en France. Puis, après deux ans de négociations, le CNRS est entré dans le capital en amenant définitivement ses brevets. C'était la condition pour qu'un deuxième tour de financement assure un investissement supplémentaire de 20 millions d'euros. Pendant d'interminables négociations, qui ont menacé l'avenir de la société, Jacques Souquet, qui pourtant fait toujours preuve d'un calme olympien, est sorti de ses gonds face à l'un de ses interlocuteurs: "Quand j'entends les arguments sans fondements que l'on m'oppose, je songe à repartir illico presto outre-Atlantique ! » La revue... À l'évidence, vous ne baissez jamais les bras.

M. F. La dernière aventure en cours, Time Reversal Communications, nous a fait revivre toutes les étapes déjà menées pour la création de Supersonic Imagine. TR Com, ce sont les téléphones du futur qui permettent d'envoyer un maximum d'informations avec un minimum d'ondes électromagnétiques. Deux sociétés de capital-risque nous ont suivis et TR Com a été finalement créée fin 2008. Peu à peu, s'est installé un climat de confiance entre le CNRS et notre laboratoire puisque les succès ont été au rendez-vous. 


\section{La revue... Si c'était à refaire ?}

M. F. Si c'était à refaire, je serais peut-être parti aux États-Unis, comme je l'avais envisagé il y a cinq ans car j'ai sans doute un profil plus adapté à l'esprit de compétition des universités américaines. J'ai maintenant appris à faire avec le système français et à éviter la plupart des écueils. D'un point de vue stratégique, j'ai maintenant une vision plus globale et précise de la façon de mener les choses en France. En allant aux ÉtatsUnis, j'aurais eu beaucoup plus de moyens et de financement. En revanche, je pense que nos étudiants français sont remarquables et tout particulièrement ceux de l'ESPCI. Je ne sais pas si j'aurais trouvé autant de brillants candidats en thèse aux États-Unis! En outre, le système du CNRS m'a permis de garder auprès de moi des chercheurs très brillants de façon permanente, ce qui aurait été plus difficile outre-Atlantique.

\section{La revue... Votre dernier projet.}

M. F. La création à partir de l'ESPCI et du CNRS d'un institut très pluridisciplinaire : l'institut Langevin travaillant sur les ondes et les images en tout genre. (On lui souhaite bonne chance, Ndlr)

Mathias Fink. (C) CNRS Photothèque/F. Jannin

\section{NOTES}

1. «[...] une recherche scientifique de qualité, mais qui débouche trop rarement ou trop tardivement sur des applications industrielles compétitives, une mobilité insuffisante des chercheurs vers l'économie, un faible dynamisme dans la création d'entreprises innovantes, la rareté des financements en capital-risque."

\section{RÉSUMÉS}

Jouant un rôle toujours croissant dans l'orientation et la géographie de l'économie comme dans la compétition entre les entreprises et entre les nations, les scientifiques participent de façon directe ou indirecte à des dynamiques collectives d'innovation et de transfert technologique. Mathias Fink nous fait revivre son parcours parfois semé d'embûches, mais toujours riche d'enseignements. 


\section{AUTEUR}

\section{MATHIAS FINK}

Mathias Fink est physicien, professeur à l'École supérieure de physique et de chimie industrielles de la Ville de Paris et membre de l'Académie des sciences. 